\title{
La perspectiva tridimensional de la calidad del cooperado en relación a la sociedad cooperativa: del rescate de la idea de un hombre cooperativo hacia la preservación de la identidad cooperativa
}

\author{
José Eduardo de Miranda \\ Doctor en Derecho por la Universidad de Deusto, \\ Profesor e Investigador de CENEC-Osório, Coordinador del Grupo \\ de Investigación Cooperación, Ciudadanía y Medio Ambiente \\ Victória Corrêa Lima de Miranda \\ Estudiante de Derecho de CNEC-Osório, Miembro del Grupo \\ de Investigación Cooperación, Ciudadanía y Medio Ambiente
}

En homenaje al Profesor Javier Divar: un ejemplo verdadero de HOMBRE COOPERATIVO.

Sumario: I. Introducción. II. La búsqueda por una definición global de sociedad cooperativa. III. La doble calidad del miembro de la sociedad cooperativa. IV. La Alianza Cooperativa Internacional y la afirmación de los valores y principios cooperativos como reto de solidificación de la identidad del Cooperativismo. V. La clave para el cambio de la calidad del miembro en relación a la sociedad cooperativa: la perspectiva tridimensional. VI. La axiología cooperativa: entre el rescate del hombre cooperativo y la preservación de la identidad del Cooperativismo. VII. Conclusión. VIII. Bibliografía.

Resumen: El objetivo de este trabajo es analizar la actual calidad que el socio ejerce en relación a la sociedad cooperativa, partiéndose de la idea que la doble condición del miembro debe ser sustituida por una orientación tridimensional sostenida por el nuevo marco de valores y principios del Cooperativismo, indispensable para el ejercicio de la condición moral del socio de la cooperativa. Para ello se examina el modus utilizado por la ACI para enunciar la Declaración sobre la Identidad Cooperativa en el Congreso de Manchester en el año de 1995, como forma de establecer un parámetro absoluto para el res- 
cate de una noción de hombre cooperativo y de consolidación de la identidad del propio Cooperativismo.

Palabras clave: Valores y principios cooperativos; Hombre cooperativo; Identidad cooperativa.

Abstract: The purpose of this study is to analyze the current quality partner exercised in relation to the cooperative society, starting from the idea that dual member's condition must be replaced by a three-dimensional orientation held by the new framework of values and principles of cooperation, indispensable for the exercise of the moral condition of member of the cooperative. For this, the modus used by the ICA to state the Statement on the Cooperative Identity at the Congress of Manchester in the year 1995 as a way to establish an absolute parameter to the rescue of a man and cooperative notion of consolidation is examined Cooperative identity itself.

Keywords: Cooperative values and principles; Man cooperative; Cooperative identity. 


\section{Introducción}

Actualmente, algunos sectores del Cooperativismo se muestran víctimas de la agudeza del ejercicio empresarial desprovisto del manto axiológico de la cooperatividad, intrínseco a las orientaciones que conforman la necesidad de la cooperativa y del cooperado existir bajo los valores y principios que les son propios.

Hoy en día, nuevamente el sistema cooperativo enfrenta un proceso de descaste que afecta distintas ramas del Cooperativismo, y el mundo contempla reconocidas cooperativas sumergir en crisis de distintas orden que acaba por quitarles del escenario jurídico, provocando traumas sociales en el contexto de inserción de la entidad cooperativa, además de destrozar la credibilidad del movimiento cooperativo como modelo socioeconómico.

En efecto, desde el año de 1995, cuando la Alianza Cooperativa Internacional promulgó la Declaración sobre la Identidad Cooperativa, el Cooperativismo mundial buscó resucitar las iniciativas de aquellos que enaltecieron la acción cooperativa, para que los cooperativistas entendiesen que su ejercicio necesitaba estar conectado con ideales propios y vinculados a una memoria axiológica que representa la dimensión fundamental de la cultura cooperativa'.

Por ello, se acreditó que a partir de la comprensión del concepto que la $\mathrm{ACl}$ formuló para la sociedad cooperativa, los cooperativistas comprenderían que la integración de los valores y principios del Cooperativismo en el contexto de vida y del ejercicio, suyo, y de la entidad cooperativa, era necesaria para garantizar la plenitud de la identidad cooperativa y para la conformación del perfil humanitario de los miembros, haciéndoles, además de socios y usuarios de la sociedad cooperativa, verdaderos paradigmas de participación y preocupación por el ajeno, colaborando con la transformación de los fenómenos que se desarrollan en el entorno de su inserción.

Sin embargo, la historia se repite.

Hoy por hoy las cooperativas pierden el hilo identitario, y los cooperativistas se dejan envolver por intereses de orden material y talante exclusivamente económico. En la medida que las sociedades cooperativas se revisten cada vez más de un perfil nítidamente capitalista, preocupándose únicamente con técnicas que maximicen sus resultados, olvidan sus orígenes y desprecian que el inicio y el fin del Cooperativismo

1 Miranda, José Eduardo de. De la crisis de identidad al rescate de la génesis del cooperativismo, Dykinson S. L., Madrid, 2012, p. 18. 
es el hombre: su calidad de vida y su realización social, económica, moral y mismo espiritual².

Necesario, entonces, repensar no la fórmula cooperativa, pero la actitud y conducta de los cooperativistas, para que el Cooperativismo no se malgaste en el tiempo e ingrese en un nuevo contexto de crisis de identidad similar o mismo peor que la crisis que anticipó el Congreso de Manchester, del 1995.

Este es, pues, el objetivo del presente trabajo.

Se pretende enmarcar la importancia que de los valores y de los principios cooperativos como pilares de existencia del propio Cooperativismo y columnas de sustentación de las relaciones humanas que se establecen en un mundo de cambios constantes y desprecio a la naturaleza humana de vivir cooperativamente.

Esto se hace necesario para demostrar que el Cooperativismo, bajo su sustrato axiológico, podrá garantizar la preservación de la identidad cooperativa, al tiempo que rescatará la génesis del hombre cooperativo, pues ya es definitiva la lección de que «la moral cooperativa no sólo implica respeto por el hombre y buenas relaciones humanas, pero también supone una moral de la buena administración» ${ }^{3}$, indispensable para las buenas relaciones entre las gentes, y entre esas y su entorno.

\section{La búsqueda por una definición global de sociedad cooperativa}

Ya queda corriente en el ejercicio de la doctrina, que las sociedades cooperativas surgieron de unos ideales forjados en el principio del siglo XIX ${ }^{4}$, y que se conformaron, más tarde, en la pauta estatutaria de la Sociedad de los Probos Pioneros de Rochdale ${ }^{5}$.

Desde entonces, y en especial a partir del año de 1895, momento en que se organiza la estructura global del Cooperativismo, bajo el ejercicio de la Alianza Cooperativa Internacional, la definición de la sociedad cooperativa siempre se mostró un ejercicio difícil, teniendo en cuenta las múltiples áreas de conocimiento que se envuelven en el es-

2 Ibídem, p. 21.

3 LASSERRE, Geroges. El Hombre cooperativo, Intercoop, Buenos Aires, 1980, p. 67.

4 Divar, Javier: La alternativa cooperativa ante la crisis, Barcelona, Ceac, 1985, p. 82

5 CAstaño I Colomer, Josep. «Conflictos respecto a la Práctica de los Principios y Valores Cooperativos: El Caso de España», Anuario de Estudios Cooperativos del Instituto de Estudios Cooperativos de la Universidad de Deusto, 1995-1996, p. 33. 
tudio del tema de la cooperatividad, consolidando su perfil de fenómeno interdisciplinario.

Mientras las leyes cooperativas de distintos países buscan establecer la delimitación jurídica del concepto de cooperativa, la sociología y la filosofía se encargan de su aspecto más humano, de contenido axioprincipiológico, y la economía atiende su perfil mercadológico, como entidad preparada para el ejercicio de las actividades encargadas de la circulación o producción de bienes o servicios.

Con apoyo de la expresión legal, se pude enmarcar que la ley brasileña de cooperativas, por su artículo $4 .^{\circ 6}$, define la cooperativa como una sociedad de personas, con forma y naturaleza jurídica propias, de carácter civil, no sujetas a quiebras, constituidas para prestar servicios a sus asociados? ${ }^{7}$.

Por su vez, la ley española de sociedades cooperativas instituye, por su artículo $1 .^{\circ}$, que las cooperativas son sociedades:

Con capital variable y estructura y gestión democráticas, asocian, en régimen de libre adhesión y baja voluntaria, a personas que tienen intereses o necesidades socioeconómicas comunes, para cuya satisfacción y al servicio de la comunidad desarrollan actividades empresariales, imputándose los resultados económicos a los socios, una vez atendidos los fondos comunitarios, en función de la actividad cooperativizada que realizan ${ }^{8}$.

De otro modo, la intención de ofrecer una aproximación al concepto global de cooperativa determina que se recurra a la Declaración de la Identidad Cooperativa que la Alianza Cooperativa Internacional adoptó en su XXXI Congreso, celebrado en la ciudad de Manchester en septiembre de 1995. En este documento, la ACI definió la cooperativa como una asociación de personas que se unen de forma voluntaria para satisfacer sus necesidades y aspiraciones económicas, sociales y culturales en común, mediante una empresa de propiedad conjunta y gestión democrática? .

6 Miranda, José Eduardo de; Galmardo, José Henrique da Silva e VIEIRA, Paulo Gonçalves: Regime jurídico da sociedade cooperativa, Juruá, Curitiba, 2013, p. 64.

7 Es importante destacar que el párrafo único del artículo 982, del nuevo Código Civil brasileño, del 2002, dispone que las sociedades cooperativas son «sociedades simples», de manera que independiente de su objeto no absorben el aspecto de entidad empresarial.

8 ESPAÑA: Ley 27/1999, de 16 de julio. Disponible en «http://www.boletinagrario. com/dc-7, ley-sociedades-cooperativas-espana,2.html», acceso en 2015/octubre/17.

9 MiRANDA, José Eduardo de: "La financiación de las sociedades cooperativas», Boletín de la Asociación Internacional de Derecho Cooperativo, n. 37, p. 233. 
Esta definición de una cooperativa pretende ser una declaración de mínimos; no pretende ser una definición de la cooperativa «perfecta». Es amplia en su alcance, reconociendo que los socios de diferentes tipos de cooperativas estarán involucrados de forma diferente y que los socios deben tener alguna libertad en la forma de organizar sus asuntos. Se espera que esta definición sea útil para la redacción de las legislaciones, la educación de los socios y la preparación de libros de texto ${ }^{10}$.

Sin embargo, observada la incidencia de valores y principios en el contexto de la cooperatividad, la sociedad cooperativa genera un perfil sintomático de entidad diferenciada, teniendo en cuenta que su constitución no se conforma con el intuito exclusivo de resolver cuestiones de orden económicas de sus miembros, sino que busca solucionar problemas de orden moral y social de los propios asociados, desarrollando, además, una preocupación por el entorno en que se encuentra inserida y colaborando con la solución de problemas generales de las gentes de la comunidad.

Las cooperativas también abarcan metas culturales en armonía con las preocupaciones de los socios: por ejemplo, ayudar en la promoción de la cultura nacional, promover la paz, patrocinar deportes y actividades culturales, y mejorar las relaciones en la comunidad. De hecho, ayudar a proporcionar una mejor forma de vida — cultural, intelectual y espiritual- podría en el futuro convertirse en una de las formas más importantes en las que las cooperativas pueden beneficiar a sus socios y hacer una contribución a sus comunidades ${ }^{11}$.

Es así que, hoy por hoy, se puede decir que la sociedad cooperativa es una entidad de carácter asociativo, compuesta por personas que se aproximan voluntariamente para un ejercicio democrático, y tiene por objeto principal la «promoción de actividades económicas y sociales de sus miembros y la satisfacción de sus necesidades con la participación activa de los mismos» ${ }^{12}$, observando la integralidad de valores y principios que determinan su responsabilidad por el medio, atendiendo adecuadamente a las necesidades de la comunidad que la cerca.

$10 \mathrm{ACl}$. «La Declaración de la Alianza Cooperativa Internacional sobre la Identidad Cooperativa: un informe», Anuario de Estudios Cooperativos del Instituto de Estudios Cooperativos de la Universidad de Deusto, 1995-1996, p. 78.

11 ACl, 1995-96, p. 93.

12 Gadea, Enrique; SACRISTÁn, Fernando y VASSERot, Carlos Vargas: Régimen jurídico de la sociedad cooperativa del siglo XXI: realidad y propuestas de reforma, Dykinson, Madrid, 2009, p. 33. 


\section{La doble calidad del miembro de la sociedad cooperativa}

Ya a partir del sustrato conceptual ofrecido por las leyes brasileña y española de cooperativas, se puede decir que la sociedad cooperativa se diferencia de las demás entidades que actúan en el mercado en virtud de su delineación muy específica.

Mientras las sociedades de capital se ajustan a partir de la aproximación de personas que conyugan voluntades o bienes con el intuito de dividir resultados económicos proporcionalmente al capital investido, en las cooperativas la fórmula se manifiesta de manera contrapuesta, pues el motivo que conduce el envolvimiento asociativo no es el interese por la división de resultados económicos, sino la posibilidad del miembro utilizarse de la sociedad cooperativa como ente prestador de servicios necesarios a la mejoría de su condición económica y social ${ }^{13}$.

En vista de ello, surge el entendimiento de que el miembro de la sociedad cooperativa es, a la vez, su socio y su usuario, o cliente.

Teniendo en cuenta esta doble característica que reviste el socio en el núcleo de la cooperativa, es posible afirmarse que, en cuanto socio, el miembro resguarda su derecho de participar de la vida de la sociedad cooperativa, integrándose al proceso gerencial a través de la presencia activa en las asambleas, actuando directamente en las deliberaciones sociales, indistintamente.

El miembro, en cuanto socio, materializa la conformación de la voluntad social y sirve para exteriorizar los actos desarrollados por la sociedad cooperativa.

No obstante, y en virtud de que la sociedad cooperativa ser constituida para prestar servicios a sus asociados, se tiene muy clara la idea de que los miembros están legitimados a utilización de todo el aparato estructural de la entidad, manejando de acuerdo con sus intereses, y en conformidad con los estatutos, los bienes y servicios ofrecidos o prestados por la sociedad cooperativa.

A par de ello, se puede decir que la doble calidad del miembro en relación a la sociedad cooperativa es utilizada por la doctrina para justificar la superación de cualquier manifiesto de ventaja económica-individual por los socios, pues su ejercicio no se consolida en virtud de una perspectiva lucrativa, una vez que el asociado espera solamente relacionarse con la cooperativa para sacarle o que tiene para ofrecerle: servicios o bienes ${ }^{14}$.

13 Franke, Walmor: Direito das sociedades cooperativas: direito cooperativo. Ed. Universidade de São Paulo, São Paulo, 1973, p. 13.

14 Ibídem, p. 14. 


\section{La Alianza Cooperativa Internacional y la afirmación de los valores y los principios cooperativos como reto de solidificación de la identidad del Cooperativismo}

Como toda la actuación desarrollada por la $\mathrm{ACl}$, también el tema de los valores cooperativos ha sido producto de un profundo y meticuloso estudio.

En el Congreso de Estocolmo de 1988 la Alianza Cooperativa Internacional observó que a pesar de todos los cambios que el movimiento cooperativo enfrentara a lo largo de su historia, era posible demostrar que a pesar de estos cambios ${ }^{15}$ siempre ha existido un imprescindible respeto por todos los seres humanos y una indiscutible creencia en la capacidad del hombre para obrar en la búsqueda de una mejora económica y social, mediante la ayuda mutua ${ }^{16}$.

La cooperación continuaba útil para afrontar las nuevas épocas. La fórmula, como en los comienzos del Cooperativismo moderno, se encontraba bajo el abrigo de los principios que el movimiento cooperativo siempre defendió con esmero. La nueva realidad global y todas las dificultades obligaron la $\mathrm{ACl}$ a preocuparse de la necesidad o no de adaptación de los principios cooperativos vigentes al nuevo contexto socioeconómico.

En lugar de analizar directamente los principios, la Alianza encarga a su presidente, Lars Marcus, la tarea de desarrollar un informe sobre las cooperativas y sus valores básicos. Esta decisión constituye un punto de arranque para abordar la cuestión de los valores y contiene muchas sugerencias y una reflexión fundamental: «las cooperativas han perdido estatura, atracción y fuerza». Además, el referido informe también sirvió de base para la futura revisión de los principios ${ }^{17}$.

En virtud de la dificultad de elaboración de un elenco completo de los valores, Marcus se limita a elaborar un informe donde indica «algunos rasgos esenciales del comportamiento humano que influyen en la vida de la cooperativa». Para ello, define que los valores básicos de la entidad cooperativa son: la participación, la democracia, la honradez y la preocupación por los demás ${ }^{18}$.

15 FARÍAS, C. A.: «Valores de la cultura económica cooperativa», Boletín de la Asociación Internacional de Derecho Cooperativo, n. ${ }^{\circ} 36$, p. 45.

16 ALIANZA COOPERATIVA INTERNACIONAL, 1996, p. 24

17 Cracogna, D.: «Reflexiones sobre los valores y los principios cooperativos en la Alianza Cooperativa Internacional», Anuario de Estudios Cooperativos del Instituto de Estudios Cooperativos de la Universidad de Deusto, 1991, p. 105.

18 MARCUS, L.: "Co-operatives and Basic Values», ICA, XXIX Congress, Stockholm, july 1988: agenda \& reports, 1988. 
Si hasta Estocolmo se creía que los valores habían emanado de los principios, desde ese Congreso se ha consolidado la idea de que los primeros derivan de los segundos y se basan en ellos.

La novedosa interpretación derivada del Congreso de Estocolmo provocó el deseo de la $\mathrm{ACl}$ dar continuidad a la investigación del tema. Por eso, con la intención proseguir con el trabajo empezado por Marcus, la $\mathrm{ACl}$ delega a Ake Böök la responsabilidad de elaborar, un meticuloso estudio sobre la materia relacionada con los valores cooperativos $^{19}$; estudio que es presentado en el Congreso de Tokio, en 1992, y sirve de base para la Declaración de Manchester, de 1995.

La profundidad del tema y la dificultad para la enunciación, o afirmación de los valores cooperativos, determina que Ake Böök desarrolle su trabajo sobre un trípode formado por las ideas e ideales; por las prácticas y experiencias; y por los proyectos y visiones para el futuro 20 .

De acuerdo con Aranzadi, la adecuación de los valores trata de una búsqueda de lo esencial, que ha animado, anima y animará en el futuro el movimiento cooperativo.

$Y$ hay que diferencias lo que constituye lo esencial de lo que es transitorio, facultativo o accesorio. Desde esa identidad, analizar las presiones exteriores técnicas o las necesidades nuevas de la población. Si queremos que tenga atractivo, no puede aparecer con una imagen trasnochada y complicada, los cooperativistas protagonistas de una acción marginal y sin gran importancia para el porvenir de la humanidad. Al contrario, debe presentarse como una alucinante aventura humana, deseable para todos los hombres de buena voluntad, y que a la vez constituye un código de ética para todos los colaboradores del movimiento cooperativo. Este afianzamiento en los valores es hoy más necesario, cuando el móvil del interés personal ha disminuido ${ }^{21}$.

Partiendo del testimonio de la propia historia mundial, que ha demostrado que distintas acciones internas en un determinado país pueden afectar y repercutir en otro u otros, Ake Böök toma como punto de partida de suprema importancia el valor de la solidaridad global como denominador común del valor cooperativo fundamental. Este valor fundamental, identificado como la línea general de acción utilizada

19 AKe Böök, S.: Valores cooperativos num mundo de mudança, Instituto Antonio Sérgio do Sector Cooperativo - Inscoop, Lisboa: 1993, p. 9.

20 Ibídem, p. 12.

21 ARANZADI, D.: El arte de ser empresario hoy: hombres y mujeres ante el reto de dirigir empresas cooperativas en el umbral del siglo XXI, Departamento de Trabajo y Seguridad Social del Gobierno Vasco - Federación de las Cooperativas de Trabajo Asociado de Euskadi - Universidad de Deusto, Bilbao, 1992, p. 279. 
por los cooperativistas para llevar a cabo su iniciativa cooperacionista, es representativo de unos valores cooperativos tradicionales identificados como ideas fundamentales, ética fundamental y principios fundamentales 22 .

Ake Böök llama la atención sobre la importancia de no priorizar los valores fundamentales, puesto que su propia naturaleza determina que la prioridad obedezca las exigencias del contexto en que son aplicados ${ }^{23}$.

Asociado o hasta dependiente de las ideas fundamentales, el estudio confiere importancia a la ética fundamental a través de los valores intrínsecos a las peculiaridades personales del individuo cooperacionista, los cuales quedan identificados como honestidad, preocupación (por los demás); pluralismo (abordaje democrática) y constructivismo (fe en la vía cooperativa).

Por fin, respeto a los principios fundamentales o valores instrumentales, estos se confunden, de una o de otra forma, con los propios principios cooperativos que la $\mathrm{ACl}$ se ha encargado de proteger, adaptar y reformular a lo largo de la historia del cooperativismo.

Es decir, la relación entre valores y principios que hacen presentes los valores en la acción de los hombres, indica que los valores se objetivan en las conductas de forma tal que la posesión de los mismos, define y determina la conducta sin que ésta deje por ello de ser manifestación del ejercicio libre de la voluntad humana ${ }^{24}$. Tanto es así que Ake Böök propone un elenco que identifica los valores instrumentales, mencionando como tales la asociación de personas; la promoción eficiente de los miembros; la gestión democrática y participación de los miembros; la autonomía e independencia; la identidad y unidad; la educación; la distribución justa de los beneficios; y la intercooperación en nivel nacional e internacional ${ }^{25}$.

Hechas estas aclaraciones, es importante anotar que la forma más relevante de expresar los valores cooperativos tradicionales, identificados como ideas fundamentales, ética fundamental y principios fundamentales, es dándoles un contexto de aplicación que va orientado a la acción.

22 GomeZ, Laura: La alianza cooperativa internacional. Su desarrollo como institución y en especial como instrumento transformador de la sociedad, CSCE - Ekgk, Vitoria, 1998, p. 112.

23 AKE BÖÖK, 1993, P. 11.

24 Cracogna, D. "La identidad cooperativa en un mundo cambiante», Anuario de Estudios Cooperativos del Instituto de Estudios Cooperativos de la Universidad de Deusto 1993, p. 89.

25 AKE BÖÖK, 1993, p. 24. 
Por esto, como los valores que han consistido en centro de actuación de los Probos de Rochdale han permanecido más o menos inalterados a lo largo del tiempo, la Alianza utilizó el Congreso de Manchester para registrar por primera vez que «las cooperativas están basadas en los valores de la autoayuda, la autorresponsabilidad, la democracia, la igualdad, la equidad y solidaridad ${ }^{26}$.

Respeto a los valores declarados en 1995, por la $\mathrm{ACl}$, interesante anotar que Díaz entiende que tanto la Alianza como la literatura se han centrado más en establecer los valores esenciales de las cooperativas y han dejado al margen los valores que tienen sus socios, "de manera que aquellos obedecen más a una declaración definitoria sobre lo que deben ser las cooperativas que a una descripción de los valores actuales que poseen los miembros de éstas» ${ }^{27}$.

El autor justifica su posición diciendo que la $\mathrm{ACl}$ se ha ocupado de los valores «que deben guiar en su camino a las cooperativas, como si fueran entes abstractos despersonalizados de su verdadera esencia (sus socios)» ${ }^{28}$.

Sin embargo, se entiende correcto manifestar una oposición a este entendimiento, pues se cree que la declaración de los valores de la $\mathrm{ACl}$, de 1995, supera el ente societario y alcanza también a sus socios ${ }^{29}$. Y tanto es así que la Alianza se ha preocupado en reconocer que siguiendo la tradición de sus fundadores, los socios cooperativos hacen suyos los valores éticos la honestidad, la transparencia, la responsabilidad y la vocación sociales ${ }^{30}$.

A pesar de estar los valores divididos en dos grupos, la intención de la $\mathrm{ACl}$ no ha sido la de jerarquizarlos, sino agruparlos, puesto que todos parecen valores de las cooperativas y de los cooperativistas. Sin embargo, lo cierto es que el segundo grupo reúne valores éticos, que pueden ser perceptibles también en actividades de organizaciones no cooperativas, capitalistas y públicas.

El Congreso realizado en Manchester, en 1995, representa el apogeo del trabajo de la $\mathrm{ACl}$, puesto que justo en la fecha de conmemora-

26 ALIANZA COOPERATIVA INTERNACIONAL, 1996, p. 17, 35 y siguientes.

27 Díaz, F.: "Cooperativismo, valores e ideología», Anuario de Estudios Cooperativos del Instituto de Estudios Cooperativos de la Universidad de Deusto 2000, p. 192. p. 130.

28 Ibídem, p. 130.

29 SANZ JARQue, J. J.: "La función de las cooperativas en el siglo XXI: valores y principios», Anuario de Estudios Cooperativos del Instituto de Estudios Cooperativos de la Universidad de Deusto 1995, p. 356.

30 Martínez Charterina, A.: "Los valores y principios cooperativos», Revista de Estudios Cooperativos. 61, diciembre de 1995. p. 43. 
ción de su centenario, ha conseguido señalar los rasgos distintivos que sostienen la verdadera identidad de las cooperativas ${ }^{31}$, destacar la importancia y alcance actual de su desarrollo, individualizar los profundos problemas y desafíos que ellas han de plantearse en el nuevo siglo, así como enunciar los principios y ratificar los valores necesarios a la preservación o concienciación del espíritu cooperativo.

\section{La clave para el cambio de la calidad del miembro en relación a la sociedad cooperativa: la perspectiva tridimensional}

Atenta a los cambios radicales que el mundo ha sufrido en los últimos años en diferentes sectores, y consciente de la sensibilidad de una época que ha estado enmarcada por el crecimiento de la conciencia de la independencia global de la humanidad, en la búsqueda de la solución de los problemas que asolan el Planeta, la $\mathrm{ACI}$ ha reaccionado con habilidad en el sentido de clarificar y fortalecer las bases ideológicas que siempre han servido de norte del movimiento cooperativo.

La reacción se concreta en el Congreso de Manchester, donde los Cooperativistas presentan la Declaración sobre la Identidad Cooperativa, elaborada en base a la nueva formulación de los principios cooperativos y en la afirmación de los valores que deben ser seguidos a lo largo de la acción cooperativa, por las entidades y por sus miembros.

De este modo, a partir de la definición de los principios como las pautas mediante las cuales las cooperativas ponen en práctica sus valores, es necesario señalar que su interpretación como patrón no sólo de acción, sino también de evaluación, les remite a los valores, que evidencian la más absoluta esencia del espíritu cooperativo.

Es decir, "considerados como un todo, estos principios se vinculan con los valores en los que se basan y resumidos en la definición, indican lo que es singular respecto a las cooperativas, independientemente de donde se ubiquen» ${ }^{32}$.

31 Enciso Santoclides, M. y Gomez Urquijo, L.: «XXXI Congreso de la Alianza Cooperativa Internacional: Crónica de lo allí acontecido», Anuario de Estudios Cooperativos del Instituto de Estudios Cooperativos de la Universidad de Deusto 1995, p. 63.

32 MAC Pherson, I.: «Los principios cooperativos», Revista de la cooperación Internacional, n. ${ }^{\circ} 28,3$, p. 25. 
Desde Manchester, se creía que las cooperativas volverían a ser lo que siempre han sido; lo que han idealizado los precursores; y lo que han emprendido los Probos Pioneros de Rochdale.

Bajo los principios y valores que han sido enunciados en el Congreso de Conmemoración del Centenario de la Alianza, las entidades cooperativas tenían que reencontrarse con su esencia; aquella que ofrece al hombre la oportunidad de desarrollar una reflexión inmaterial, solidaria, de preocupación con su compañero y con todos aquellos vinculados a su ambiente, incluso el extra-cooperativo.

Hoy por hoy, después de reencontrada con la identidad perdida, la cooperativa debería volver a su marco de acción inicial, presente en los estatutos originales de los Probos Pioneros de Rochdale. Por esta línea, se debe mantener intocable la certeza de que su objeto mayor supera el económico, y los principios y valores cooperativos le sirven para buscar la mejora de las condiciones socio-económico-morales y espirituales del hombre.

Es así que la principal tarea de la entidad cooperativa es sembrar el entendimiento entre los ciudadanos; ubicar su ejercicio empresarial en el sentido más ventajoso al bien común; favorecer la solución de las dificultades, permitiendo nuevos caminos que conduzcan a los individuos al encuentro de la prosperidad; y participar del desarrollo educativo del ser humano, de forma que éste se vea involucrado en una existencia basada en la labor coparticipativa.

Como en los albores del siglo XIX, en la medida que se convierten en los medios necesarios para transmitir al conjunto de la sociedad valores esenciales como son la democracia, la participación, la solidaridad, la igualdad, la equidad, la responsabilidad, la transparencia y la honestidad, la cooperativa, y por supuesto, el Cooperativismo, se transforman en un paradigma social.

En todos los tiempos, el elemento común de la cooperación ha consistido siempre en su intento incansable de promover algo más que los intereses de los miembros individuales que componen la estructura empresarial cooperativa.

Por ello, se debe una y otra vez realzar que el objetivo del Cooperativismo es, ante todo, fomentar el progreso y el bienestar de la humanidad. Es por esto que el trabajo que culminó con la Declaración sobre la Identidad Cooperativa, editada en el Congreso de Manchester, de 1995, recupera la idea casi olvidada de que el Cooperativismo no es un fin en sí mismo; que, las cooperativas no tienen el objetivo único de asegurar su supervivencia, sino que su meta es vivir, para proporcionar que personas vivan de una forma mejor. 
Así que la $\mathrm{ACl}$ revuelve el sentimiento cooperativo, y hace que nuevamente los cooperativistas perciban que su función es responder a las cuestiones de la humanidad16533, del mundo, y del mañana, de forma que permita a los hombres ubicar su existencia en un contexto diferente, superior.

No se va a decir ahora que la Declaración de la $\mathrm{ACl}$ sobre la Identidad Cooperativa ha sido un hecho innovador, pero se debe enmarcar una y otra vez que ha sido un logro histórico, que antes de más nada ha conseguido colocar nuevamente al Cooperativismo bien consigo mismo. Es decir, la Declaración, o afirmación de los valores y la nueva formulación de los principios cooperativos han servido para reeditar la idea, la filosofía, la importancia del factor humano. Y es justamente este factor el que genera una tendencia interna, entre los miembros de la entidad cooperativa, en el sentido de desencadenar unas relaciones que hacen de ella no simplemente una empresa, en su aspecto económico, sino también una asociación, una asociación entre hombres que además de preocuparse por la satisfacción de sus necesidades, no se olvidan de la preocupación por las necesidades de los demás.

Los valores cooperativos, y los principios, representando las pautas para la puesta en práctica de aquellos, dejan otra vez el Cooperativismo preparado para enfrentar sus objetivos fundamentales: la insurrección contra las necesidades y la insurrección contra la inmoralidad, la inhumanidad, la injusticia económica y social, contra el olvido.

Este moverse contra, deja patente que las cooperativas, como entidades socio-económicas, se fundan básicamente sobre valores determinantes de la autoayuda, la responsabilidad, la democracia, la igualdad, la equidad, la solidaridad, la honestidad, la transparencia, y la vocación social. Valores estos que son puestos en práctica por unos principios que determinan que los fines de la Cooperación se extienden más allá de la promoción de los intereses económicos individuales de los cooperativistas, para contribuir con los valores sociales y morales de una sociedad que necesita el amparo del hombre cooperativo.

Los valores y principios cooperativos son condicionantes de una actuación humana-empresarial, volcada a la dignidad y a la justicia, que determina un sistema de deberes y acciones que entidad e individuos han de llevar a cabo: uno en pro del otro, y ambos a favor de los demás, sin perjuicio de los intereses particulares del miembro y de la empresa.

33 Rosembus, T.: «El valor del socio en la cultura cooperativa», Boletín de la Asociación Internacional de Derecho Cooperativo, n. ${ }^{\circ} 36$, p. 15. 
De un modo muy claro, la interpretación de los valores y principios cooperativos revela la imagen de que las cooperativas, de nuevo, se encuentran a merced de una tarea trascendental que implica la acción de comprender y respetar las necesidades humanas; de atender a las necesidades de iniciar y ayudar a los individuos a seguir el camino del progreso personal, de la promoción, de la expansión y del perfeccionamiento del factor humano de cada persona.

Esta es la moral cooperativa, la clave para el cambio de la calidad del socio delante da la sociedad cooperativa. Esta es la alternativa para el estabelecimiento de una calidad tridimensional del socio en relación a la cooperativa...

Una vez comprendida la esencia axiológica de la cooperación, a partir del entendimiento del significado de los valores y principios cooperativos, es necesario superarse el aspecto doble, o dupla calidad que el miembro tiene delante de la sociedad cooperativa, pasándose conformar un ejercicio tridimensional, pues, teniendo en vista la transcendencia de los propios principios y valores en el marco de identidad de la cooperativa, el miembro, además de socio y usuario también desarrolla un ejercicio moral, o una labor ética, por la cual expresa un referencial a ser seguido en el entorno de inserción de la sociedad cooperativa.

Es a partir de su relacionamiento con el mundo que el socio de la sociedad cooperativa muestra a todos los ciudadanos la importancia de la puesta en práctica de las virtudes políticas, como la tolerancia, el respeto mutuo, la reciprocidad, y la idea de equidad y civilidad. «La enseñanza razonable debe fortalecer las formas de pensar y sentir que sostienen la cooperación social equitativa entre los hombres que se deben considerar libres e iguales» ${ }^{34}$

La reflexión sobre estas virtudes políticas deja claro que es el miembro de la sociedad cooperativa el verdadero responsable, en el universo cooperativo, por la educación y formación de futuras generaciones dentro del espíritu de la cooperación dentro de un espíritu de fraternidad, de igualdad, de solidaridad y de justicia.

Es justo en esta dirección que se encamina la conducta imprescindible para el aprendizaje que tenga como meta fundamental del hombre construir una sociedad basada en principios éticos sostenidos por una visión universalista de valores humanos y jamás por una responsabilidad moral individualista y neopragmatista.

34 Silva, S. R.: Formação moral em Rawls, Editora Alínea, Campinas, 2003, p. 125 
La calidad tridimensional, además del aspecto de socio y usuario que el miembro tiene en relación a la sociedad cooperativa, se reviste también de su compromiso mora, de no perder la génesis axiológica en relación al ejercicio de la propia cooperativa, y en especial en correspondencia a manera por la cual se relaciona con el mundo y con sus ajenos.

\section{La axiología cooperativa: entre el rescate del hombre cooperativo y la preservación de la identidad del Cooperativismo}

La sociedad cooperativa corresponde a una expresión técnica jurídica de un radio social dilatado. Su clasificación y acogimiento no influye sobre la clase social que la asume ni, en principio, la reacción asociativa dispersa efectos más allá del fin común que los socios se proponen desarrollar.

Sin embargo, la sociedad cooperativa siempre ha constituido un medio de intervención, un instrumento de realización del derecho de participación del ciudadano en la vida económica, social y cultural de su entorno.

La forma en que se articula la sociedad cooperativa la hace, en sentido técnico jurídico, una entidad basada en la participación personal de sus socios. Esta participación se establece a partir de una jerarquía de fines y pautas de las acciones, que encuentra su mejor acomodo en la definición de valores y la revisión de los principios.

Esa relación entre valores y principios que hacen presentes los valores en la acción de los hombres, indica que los valores se objetivan en las conductas de forma tal que la posesión de los mismos, a veces se les ha considerado como virtudes, define y determina la conducta sin que ésta deje por ello de ser la manifestación del ejercicio libre de la voluntad humana ${ }^{35}$.

La verdad es que la $\mathrm{ACl}$, a través del Congreso de Manchester, ha dejado claro que el existir de la sociedad cooperativa está condicionado a la interpretación de los principios no solo como pautas de acción, sino de promoción de valores.

35 Martinez Charterina, A.: «Los valores y principios cooperativos», Revista de Estudios Cooperativos, n. ${ }^{\circ} 61$, p. 38. 
Por esto, y considerando que las cooperativas ya están consagradas como fórmula que permite a los menos agraciados una alternativa para la conjugación de todas las ventajas de la vida privada, se entiende en Aranzadi que las cooperativas, y por supuesto, el Cooperativismo, debe ser «el portaestandarte de unos valores que todos los hombres de buena voluntad han de asumir y que deben ser el norte de la visión ética y humanizadora» ${ }^{36}$.

De esta manera, resta muy claro que los valores cooperativos se deducen de una relación co-operativa entre el hombre y la sociedad, es decir, de una concepción antropológica del hombre y de sus relaciones sociales. Es aquí, cuando se conforma el modus comportamental de un hombre que se integra efectivamente a su medio, se tiene rescatado el perfil del hombre cooperativo; el hombre que busca, de verdad, solucionar sus problemas personales, más no se aleja de los problemas del medio y de sus comunes.]

En la medida que se establece este perfil de postura actitudinal, el ejercicio de la cooperativa se manifiesta de acuerdo con el fundamento del Cooperativismo, teniéndose garantizada la preservación de su identidad.

Por ello es necesario exaltar más una vez que las aspiraciones morales del Cooperativismo se combinan con las normas estatutarias de Rochdale porque esta cooperativa ha estado al servicio de los intereses de sus miembros, por su singular condición de socio, y al mismo tiempo ha buscado atender las carencias de la comunidad, por la razón de que antes de nada los Probos vislumbraban la posibilidad de promover cambios en el orden social.

\section{A título de conclusión}

Nadie más duda que la Declaración de la Identidad Cooperativa, de la Alianza Cooperativa Internacional, surgiera con el propósito de reorganizar el ejercicio de las sociedades cooperativas, involucrando sus miembros a la esencia de la cooperatividad, demarcada por valores y principios que conforma el verdadero espíritu del Cooperativismo.

36 ArAnZAdi, D.: Los valores cooperativos en el umbral del año 2000, Anuario de Estudios Cooperativos del Instituto de Estudios Cooperativos de la Universidad de Deusto, 1993, p. 128. 
El aspecto profundamente humano de la cooperación consiste en una superioridad que complica las cosas pero cuyo valor es inestimable. En efecto, se observa que en algunos pueblos, en algunos medios donde se había formado un poderoso espíritu cívico o que tenían buenos hábitos democráticos, o inclinados hacia la libre asociación o cuyo sentimiento de responsabilidad era más profundo, las cooperativas tuvieron éxito; allí se forjó la calidad humana necesaria ${ }^{37}$.

De esta forma, y en atención a las primeras sociedades que han sido constituidas, debiese subrayar que las cooperativas se destacan, sobre todo, por representar entidades creadas por personas que tienen dificultades comunes y buscan, a través de la ayuda mutua, la satisfacción o solución de sus respectivos problemas.

Genéricamente, se puede decir que las cooperativas han sido creadas para permitir a los hombres el logro de una justa distribución de la riqueza social; la igualdad de oportunidades para todos; evitar que los hombres se exploten entre sí; imposibilitar el desarrollo de clases parasitarias que vivan a costa de los demás; y frenar el predominio del capital sobre la actividad humana.

Refiriéndose al tema, Lassere certifica que el descubrimiento de la idea cooperativa halla a menudo el hombre de hoy a un terreno más o menos preparado.

En términos más amplios pueden hallar en el fondo de la persona humana - muchas veces oculta bajo varias capas que obligan a cavar hondo para hallarla - la necesidad de superación, de un objetivo en la vida mayor que dicha necesidad. Le brindan una ocasión y un medio para actuar contra la miseria y la explotación, para contribuir a edificar una sociedad nueva y mejor ${ }^{38}$.

En este sentido, es necesario subrayar que antes del desarrollo de la doctrina cooperativa y de la elaboración de leyes específicas sobre cooperativas, el mundo ya conocía asociaciones cooperacionistas, o la cooperación en su más puro significado.

Alejadas de cualquier estructura reglamentaria, las diferentes formas de cooperación resultaban de la unión libre, espontánea y voluntaria de personas que, con fines sociales y económicos, persiguen el propio bien y el de la comunidad.

37 LASSERRE, 1980, p. 13.

38 Ibídem, p. 92. 
La cooperación de antaño alcanza sus objetivos independientemente de la existencia de reglas particulares que apuntasen al desarrollo de sus actividades.

Por ello, e independiente de los avances de la legislación, la doctrina no soterrar las raíces de las cooperativas y desfalcar el Cooperativismo de su aspecto social, antes que económico, humano, más allá que material.

Hay, por tanto, que sobrellevarse la conciencia del universo cooperativo que las extravagancias inherentes a las distintas ramas del Cooperativismo son predisposiciones malas que el día menos pensado someterán los cooperativistas a una nueva crisis de identidad que, quizás, le afecte de tal manera su estructura y funcionalidad que pierda definitivamente su razón de ser, su marca de representatividad como la alternativa que, más allá de permitir el desarrollo socioeconómico del hombre, le permite una evolución moral y mismo espiritual.

Es así que, mientras se ve la actuación de algunos gestores de cooperativas que preponderan la importancia de la juridicidad, la jerarquía de la forma y del material antes del fin más grande del Cooperativismo, el hombre, se debe decir, en definitivo, que el movimiento cooperativo actúa sobre hombres, para hombres y em razón de los hombres.

Se hace necesario, así, que los cooperativistas entiendan que además de su ejercicio de socio y miembro de la sociedad cooperativa, tienen ellos una responsabilidad moral, de difundir la cultura cooperativa y materializar el espíritu cooperativo a través de un ejercicio de humanidad colaborativa con el entorno y con el ajeno.

La tridimensionalidad de la calidad del miembro en relación a la sociedad cooperativa consolida el valor supremo del Cooperativismo, que pone el hombre en el blanco de inicio y fin de sus objetivos. Por ello, hay que gritarse siempre, que, si en cualquier doctrina, una hipótesis no es válida hasta que ha sido demostrada experimentalmente, en las doctrinas sociales es imprescindible el experimento con los hombres, la acción social 39 .

Por tanto, parece evidente, y muchos no quieren ver, que uno jamás podrá hacer doctrina cooperativa confortablemente acomodado en un despacho aséptico, desde una cátedra elevada, o en el peldaño de un cargo o función burocrática, ejecutiva, lejos de los murmullos y las singularidades del ejercicio humano de ser gente.

Nadie hará doctrina cooperativa o será un verdadero cooperativista dictando reglas ajenas de la axiología del Cooperativismo; ejerciendo

39 Ibídem, p. 21 
en las sociedades cooperativas puestos ejecutivos que le mantenga lejos de las necesidades de la gente, distante del espíritu cooperativo, y próximo únicamente del ánimo especulativo, que resuelve a penas sus ambiciones, con desprecio a los demás, con desprecio al mundo, y con desprecio al propio Cooperativismo.

\section{Bibliografía}

$\mathrm{ACl}$. «La Declaración de la Alianza Cooperativa Internacional sobre la Identidad Cooperativa: un informe», Anuario de Estudios Cooperativos del Instituto de Estudios Cooperativos de la Universidad de Deusto, 1995-1996.

AKE Böök, S.: Valores cooperativos num mundo de mudança, Instituto Antonio Sérgio do Sector Cooperativo - Inscoop, Lisboa: 1993.

ARANZADI, Dionísio: El arte de ser empresario hoy: hombres y mujeres ante el reto de dirigir empresas cooperativas en el umbral del siglo XXI, Departamento de Trabajo y Seguridad Social del Gobierno Vasco - Federación de las Cooperativas de Trabajo Asociado de Euskadi - Universidad de Deusto, Bilbao, 1992.

Los valores cooperativos en el umbral del año 2000, Anuario de Estudios Cooperativos del Instituto de Estudios Cooperativos de la Universidad de Deusto, 1993.

CASTAÑo I Colomer, Josep: «Conflictos respecto a la Práctica de los Principios y Valores Cooperativos: El Caso de España», Anuario de Estudios Cooperativos del Instituto de Estudios Cooperativos de la Universidad de Deusto, 1995-1996.

CRACOGNA, Dante: «Reflexiones sobre los valores y los principios cooperativos en la Alianza Cooperativa Internacional», Anuario de Estudios Cooperativos del Instituto de Estudios Cooperativos de la Universidad de Deusto, 1991.

«La identidad cooperativa en un mundo cambiante», Anuario de Estudios Cooperativos del Instituto de Estudios Cooperativos de la Universidad de Deusto 1993.

Díaz, F.: "Cooperativismo, valores e ideología», Anuario de Estudios Cooperativos del Instituto de Estudios Cooperativos de la Universidad de Deusto 2000.

DIVAR, Javier: La alternativa cooperativa ante la crisis, Barcelona, Ceac, 1985.

Enciso SAntoclides, M. y Gomez UrquiJo, L.: «XXXI Congreso de la Alianza Cooperativa Internacional: Crónica de lo allí acontecido», Anuario de Estudios Cooperativos del Instituto de Estudios Cooperativos de la Universidad de Deusto 1995

EspañA: Ley 27/1999, de 16 de julio. Disponible en «http://www.boletinagrario. com/dc-7, ley-sociedades-cooperativas-espana,2.html», acceso en 2015/octubre/17. 
Farías, C. A.: «Valores de la cultura económica cooperativa», Boletín de la Asociación Internacional de Derecho Cooperativo, n. ${ }^{\circ} 36$

FRANKE, Walmor: Direito das sociedades cooperativas: direito cooperativo. Ed. Universidade de São Paulo, São Paulo, 1973.

GADEA, Enrique; SACRISTÁN, Fernando y VASSEROT, Carlos Vargas: Régimen jurídico de la sociedad cooperativa del siglo xxI: realidad y propuestas de reforma, Dykinson, Madrid, 2009.

JuLIÁ IGUAL, J. F. y GalLego SeVILLA, L. P.: «Principios cooperativos y legislación de la sociedad cooperativa española. El camino hacia el fortalecimiento de su carácter empresarial», Revista de Estudios Cooperativos, n. ${ }^{\circ} 70$.

LASSERRE, Georges: El Hombre cooperativo, Intercoop, Buenos Aires, 1980.

MAC PHeRSON, I.: «Los principios cooperativos», Revista de la cooperación Internacional, n. ${ }^{\circ} 28,3$.

MARCUS, L.: "Co-operatives and Basic Values», ICA, XXIX Congress, Stockholm, july 1988: agenda \& reports, 1988.

MARTINEZ ChARTERINA, Alejandro: "Los valores y principios cooperativos», Revista de Estudios Cooperativos, n. ${ }^{\circ} 61$, p. 38.

Miranda, José Eduardo de; Galmardo, José Henrique da Silva e Vieira, Paulo Gonçalves: Regime jurídico da sociedade cooperativa, Juruá, Curitiba, 2013.

MIRANDA, José Eduardo de: "La financiación de las sociedades cooperativas», Boletín de la Asociación Internacional de Derecho Cooperativo, n. ${ }^{\circ} 37$.

De la crisis de identidad al rescate de la génesis del cooperativismo, Dykinson S. L., Madrid, 2012.

Rosembus, Túlio: «El valor del socio en la cultura cooperativa», Boletín de la Asociación Internacional de Derecho Cooperativo, n. ${ }^{\circ} 36$.

SANZ JARQUE, J. J.: «La función de las cooperativas en el siglo XXI: valores y principios», Anuario de Estudios Cooperativos del Instituto de Estudios Cooperativos de la Universidad de Deusto 1995.

SILVA, S. R.: Formação moral em Rawls, Editora Alínea, Campinas, 2003. 